Claremont Colleges

Scholarship@ Claremont

All HMC Faculty Publications and Research

HMC Faculty Scholarship

$1-1-2007$

\title{
Control of Strong-Laser-Field Coupling to Electrons in Solid Targets with Wavelength-Scale Spheres
}

\section{H. A. Sumeruk}

University of Texas at Austin

S. Kneip

University of Texas at Austin

D. R. Symes

University of Texas at Austin

I. V. Churina

University of Texas at Austin

A. V. Belolipetski

University of Texas at Austin

See next page for additional authors

\section{Recommended Citation}

H. A. Sumeruk, S. Kneip, D. R. Symes, I. V. Churina, A. V. Belolipetski, T. D. Donnelly, and T. Ditmire, "Control of Strong-Laser-Field Coupling to Electrons in Solid Targets with Wavelength-Scale Spheres," Phys. Rev. Lett., 98, 045001 (2007). doi: 10.1103/

PhysRevLett.98.045001

This Article is brought to you for free and open access by the HMC Faculty Scholarship at Scholarship @ Claremont. It has been accepted for inclusion in All HMC Faculty Publications and Research by an authorized administrator of Scholarship @ Claremont. For more information, please contact scholarship@cuc.claremont.edu. 
Authors

H. A. Sumeruk, S. Kneip, D. R. Symes, I. V. Churina, A. V. Belolipetski, Thomas D. Donnelly, and T. Ditmire 


\title{
Control of Strong-Laser-Field Coupling to Electrons in Solid Targets with Wavelength-Scale Spheres
}

\author{
H. A. Sumeruk, ${ }^{1}$ S. Kneip, ${ }^{1}$ D. R. Symes, ${ }^{1}$ I. V. Churina, ${ }^{1}$ A. V. Belolipetski, ${ }^{1}$ T. D. Donnelly, ${ }^{2}$ and T. Ditmire ${ }^{1}$ \\ ${ }^{1}$ Texas Center for High Intensity Laser Science, Dept. of Physics, The University of Texas at Austin, Austin, Texas 78712, USA \\ ${ }^{2}$ *Physics Department, Harvey Mudd College, Claremont, California, USA
}

(Received 2 June 2006; published 23 January 2007)

\begin{abstract}
Irradiation of a planar solid by an intense laser pulse leads to fast electron acceleration and hard x-ray production. We have investigated whether this high field production of fast electrons can be controlled by introducing dielectric spheres of well-defined size on the target surface. We find that the presence of spheres with a diameter slightly larger than half the laser wavelength leads to Mie enhancements of the laser field which, accompanied by multipass stochastic heating of the electrons, leads to significantly enhanced hard x-ray yield and temperature.
\end{abstract}

DOI: 10.1103/PhysRevLett.98.045001

PACS numbers: 52.38. $-\mathrm{r}$

When an intense ultrafast laser irradiates a solid target, plasma instabilities and the laser field itself generate hot electrons which produce ultrafast bursts of $\mathrm{x}$ rays. These have been exploited for various time-resolved $\mathrm{x}$-ray probing applications [1]. The mechanisms for this hot electron generation include stimulated scattering in the under-dense plasma in front of the target and resonance absorption near the critical density surface [2]. When very short pulses are employed (sub-100 fs), and the scale length of the plasma is much shorter than a wavelength, vacuum heating, often called Brunel absorption, is the dominant mechanism for hot electron production [3]. Because vacuum heating is sensitive to the structure of the laser field at the target surface, this electron heating mechanism can be controlled by introducing wavelength-scale structures to the laser target; these alter the local boundary conditions which constrain the field. Controlling the laser field in this manner can lead to more efficient and higher temperature electron and hard x-ray production. Early studies showed that targets coated with $10 \mathrm{~nm}$-scale structures (e.g., gold clusters [4], nickel "velvet" [5], or Al colloids [6]) led to enhanced soft x-ray (i.e., sub-keV) emission. The explanation in these experiments is that these roughened targets simply absorb more laser energy than polished flat targets leading to a hotter thermal plasma at the target surface. More recently, hard x-ray production has been investigated by Rajeev et al. who found that hard x-ray yields were enhanced when targets roughened on the $0.1 \mu \mathrm{m}$ scale [7] or when covered with nanoscale particles scale were irradiated [8]. Similar field-enhanced absorption by gaseous targets of nanometer-scale clusters has also been well documented $[9,10]$.

Most of these studies have been carried out with targets in which the structures are a few nanometers and are, therefore, much smaller than the wavelength of light. It well known that particles with sizes comparable to the light wavelength can lead to very large enhancements of the laser field $(\sim 10-100$ times the vacuum field for conduct- ing particles) through so-called Mie resonances [11]. Because of the potential for inducing these field enhancements on the surface, a target coated with appropriately chosen spheres should lead to much higher hot electron yields and temperatures than simple planar solid targets. Such field enhancements have already been shown to affect the explosion of intensely irradiated micron-scale droplets [12]. Micron-scale particle targets are also interesting because stochastic heating of electrons from multiple scattering off the particle surface is possible [13-15]. While Brunel heating of electrons from planar solids is the result of a single return to the target surface and acceleration in the field by a single half cycle, irradiation of a plasma particle can result in multiple passes and greater electron energy. This phenomenon was inferred in experiments in which micron-scale water droplets were irradiated by an intense $40 \mathrm{fs}$ laser [16]. This experiment employed a target composed of a broad range of particle sizes, so the effects of Mie resonances for particular particle sizes could not be manipulated or observed.

In this Letter, we report on experiments in which an intense, high contrast $400 \mathrm{~nm}$ pulse with under $100 \mathrm{fs}$ duration irradiates two dimensional arrays of polystyrene particles of single, well-defined sizes deposited on the surface of a planar solid. Unlike previous studies, this approach has allowed systematic study of structures size on field enhancements and consequent hot electron generation. We find that by varying the size of these particles, an optimum particle size leads to enhanced hot electron production and higher electron temperature. We attribute this effect, which is maximum for particles with $\sim 260 \mathrm{~nm}$ diameter, to electric field enhancements at the surface of the particle and multiple transit stochastic heating of the electrons.

To perform this study, we employed the THOR laser at the University of Texas, a laser which delivers 35 fs pulses at $800 \mathrm{~nm}$ with energy up to $0.8 \mathrm{~J}$. We found that when we employed the $800 \mathrm{~nm}$ pulses to irradiate a solid, amplified 
spontaneous emission and picosecond time scale prepulses led to substantial plasma expansion prior to the arrival of the main pulse [17]. To improve pulse contrast, we frequency-doubled the laser to $400 \mathrm{~nm}$ and filtered away the $800 \mathrm{~nm}$ light with dichroic mirrors. Based on high dynamic third order autocorrelator measurements of the prepulses on the fundamental pulse, we estimated that the prepulse level was not greater than $10^{-7}$ of the main pulse when the fundamental was frequency-doubled. We conducted hot electron production measurements with $p$-polarized pulses on planar silica targets as a function of irradiation angle and found a maximum in hard $\mathrm{x}$-ray production at $55^{\circ}$ [17], the exact angle that is maximum for Brunel absorption [3]. This indicated that plasma expansion of well under the electron oscillation amplitude $\left(\lambda_{\text {osc }} \sim 15 \mathrm{~nm}\right)$ occurred and that our pulses interact with well-defined unexpanded spheres. We focused $12 \mathrm{~mJ}$ of $400 \mathrm{~nm}$ light with an $f / 3$ parabola to an intensity of around $2 \times 10^{17} \mathrm{~W} / \mathrm{cm}^{2}$.

We used the $400 \mathrm{~nm}$ pulses to irradiate solids with monolayers of polystyrene particles deposited on the surface. The polystyrene spheres were of single size with size dispersion of $\leq 3 \%$. These spheres, which are delivered suspended in water solution, were deposited on the surface of either polished silica substrates or silicon wafers and usually formed hexagonally-packed single layers, as illustrated in Fig. 1. We coated targets with spheres of sizes ranging from $100 \mathrm{~nm}$ to $2.9 \mu \mathrm{m}$ diameter.

We examined x-ray production over the 1 to $100 \mathrm{keV}$ energy range from both flat and sphere-coated targets. A crystal spectrometer in Von Hamos configuration was employed to examine $\mathrm{K}$-alpha emission from the underlying silicon at 7.1 angstroms $(1.7 \mathrm{keV})$. Hard $\mathrm{x}$-ray detectors with $\mathrm{NaI}$ crystals coupled to photomultiplier tubes were used to measure bremsstrahlung x-ray yield from 20$80 \mathrm{keV}$. We deployed an array of these detectors with six different cutoff filters (composed of $\mathrm{Fe}$ and $\mathrm{Cu}$ slabs of differing thickness). By correcting for the photon energy dependent detection efficiencies and by assuming an exponential spectrum of single temperature, we could ascertain an x-ray temperature and hence an electron temperature.

We observe an increase in the $\mathrm{x}$-ray yield when dielectric spheres are deposited on a target surface, an increase whose magnitude becomes more dramatic with harder X-rays. Figure 2(a) illustrates Si K-alpha spectra measured from planar, polished targets and from targets coated with a layer of $260 \mathrm{~nm}$ spheres. We measured x-ray yield from planar targets with both $p$ - and s-polarization as a function of angle. At all irradiation angles and polarizations, the $\mathrm{x}$-ray yield from targets with small $(<500 \mathrm{~nm})$ spheres exhibited K-alpha yields which were higher than any irradiation configuration (at equal laser intensity) on planar solids. We also compared $\mathrm{x}$-ray yield when a thin poly(methyl methacrylate) layer (very similar in atomic composition and density to the polystyrene spheres) was deposited on the target surface or when the polished surface was roughened on the sub-micron-scale, similar to

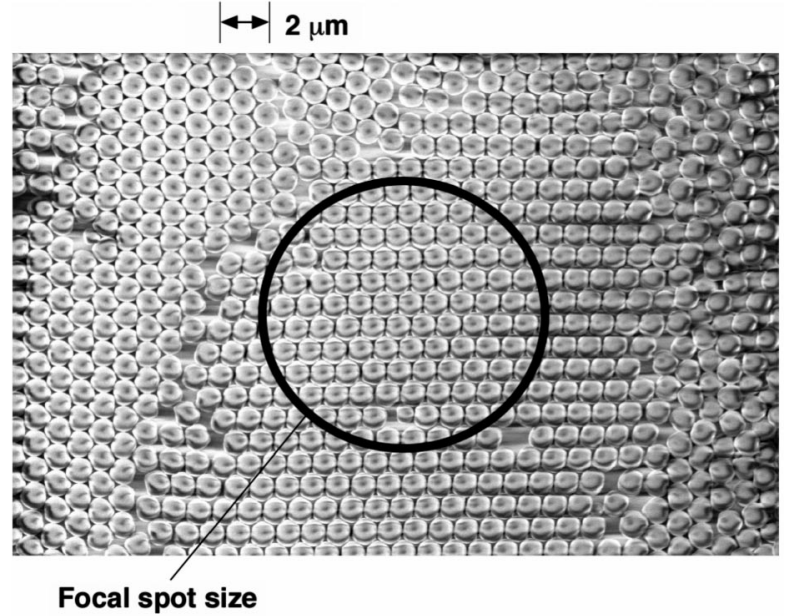

FIG. 1. Scanning electron microscope image of $1 \mu \mathrm{m}$ diameter polystyrene spheres arrayed on a $\mathrm{Cu}$ substrate. The apparent dimples on the spheres are an artifact of the SEM measurement.

Ref. [7] (the data from roughened targets are also plotted in Fig. 2(a)). In all cases, yield from sphere-coated targets was the highest. This indicates that the enhancement could not simply be attributed to resonance absorption or vacuum heating at some optimum angle and must arise specifically from the geometry of the spherical targets. The x-ray enhancement was even more pronounced in the hard $\mathrm{x}$-ray $(>20 \mathrm{keV})$ spectra. We observed an enhancement by a factor of 8 in yield of x-rays $>22 \mathrm{keV}$ from targets coated by $260 \mathrm{~nm}$ spheres when compared to yield from planar targets irradiated at $55^{\circ}$ incidence with $p$-polarization. Figure 2(b) plots $\mathrm{x}$-ray yield measured through filters with various $1 / e^{2}$ cutoff energies for a planar silica target and from targets coated with $100 \mathrm{~nm}$ and $260 \mathrm{~nm}$ spheres. A clear increase in x-ray yield is seen, and a hardening of the $\mathrm{x}$-ray temperature is also evident from the slope of the signals. A minimal difference is observed in yield and temperature when $260 \mathrm{~nm}$ spheres are irradiated at different angles (in stark contrast to planar solids).

We observe a strong dependence of $\mathrm{x}$-ray production with the sphere size employed. Figure 3(a) plots Si K-alpha yield as a function of sphere size coating $\mathrm{Si}$ substrates. Hard x-ray yield at $22 \mathrm{keV}$ and $75 \mathrm{keV}$ as a function of sphere size coating silica substrates is illustrated in Fig. 3(b). In both cases, we see a peak in the x-ray yield when $260 \mathrm{~nm}$ spheres coat the target surface. K-alpha yield with large, $2.9 \mu \mathrm{m}$ spheres is roughly comparable to yield measured from planar Si targets (irradiated at 45 degrees in $p$-polarization) while yield with $260 \mathrm{~nm}$ spheres is 5 times that of either. For hard $x$ rays, the presence of spheres on the target surface enhances yield over planar targets; however, the yield is highest when $260 \mathrm{~nm}$ spheres are employed. We also examined hot electron temperature as a function of sphere size (determined from hard x-ray temperature assuming photon production by thin target bremsstrahlung [18]). Figure 3(c) shows that there is a peaking of $\mathrm{x}$-ray temperature when spheres of diameter of 260 to 

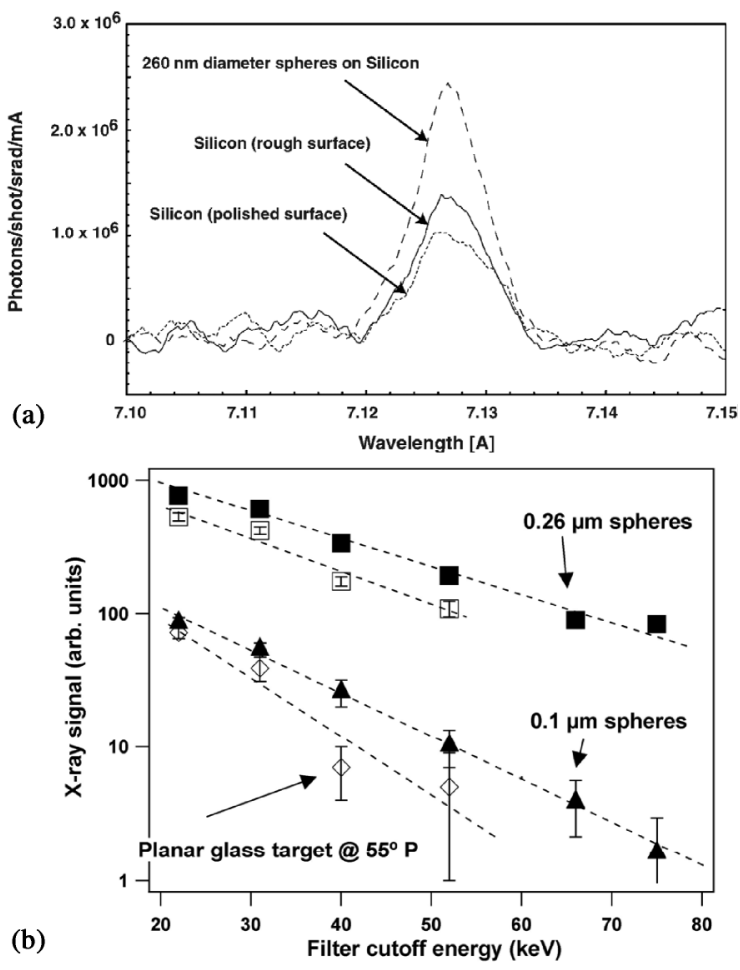

FIG. 2. a) Si K-alpha spectra integrated over 200 shots. The spectrum from a $\mathrm{Si}$ wafer coated with $260 \mathrm{~nm}$ spheres is compared to spectra from polished flat $\mathrm{Si}$ surfaces and from $\mathrm{Si}$ surfaces with sub-micron-scale random roughness collected when the Si targets were irradiated at 45 degrees with $p$-polarized $400 \mathrm{~nm}$ light. b) Hard $\mathrm{x}$-ray signal through various filters as a function of the $1 / e^{2}$ cutoff of each filter. The x-ray spectra from silica targets coated with $260 \mathrm{~nm}$ spheres (open squares from $55^{\circ}$ irradiation and closed squares from normal irradiation) are compared to spectra derived from targets coated with $100 \mathrm{~nm}$ spheres (normal incidence) and from flat targets (irradiated at $55^{\circ}$ with $p$-polarization). Error bars were determined from the scatter in data from multiple laser shots.

$1000 \mathrm{~nm}$ are irradiated and that the temperature is significantly higher than from a planar solid.

To ascertain the importance of Mie resonances in this hot electron production, we have calculated the field structure around arrays of plasma spheres. This we do using the computational approach of Mackowski and Mishchenko, which solves Maxwell's equations around and in an ensemble of spheres of given dielectric constant, $\epsilon$ [19]. We assume a Drude model for the plasma $\epsilon$ and treat the plasma sphere as being triply ionized (though this choice makes little difference). The calculated square of the electric field compared to the field in vacuum around a hexagonal array of $260 \mathrm{~nm}$ spheres irradiated with $400 \mathrm{~nm}$ light is illustrated in Fig. 4(a). Much of the sphere surface can see effective intensity over 10 times the vacuum intensity, and small areas see 30 times that of the vacuum intensity. This will lead to greater extraction of electrons and greater acceleration to higher velocity. The extent of the calculated field enhancement on a sphere surface as a function of sphere size is illustrated in Fig. 4(b). There is an
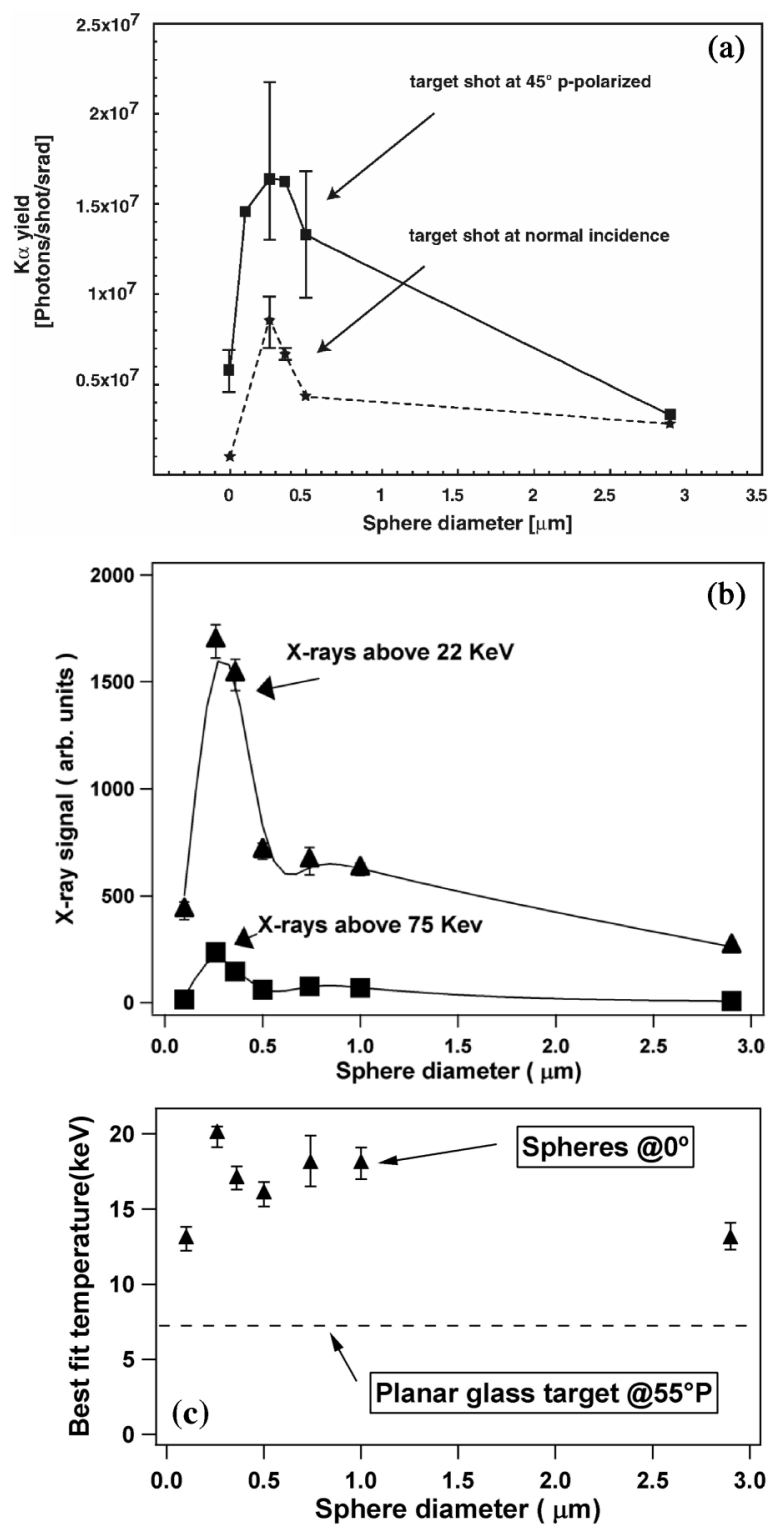

FIG. 3. a) Si K-alpha yield as a function of sphere size coated on Si wafers. b) Hard x-ray yield through two different filters as a function of sphere size. c) Hot electron temperature derived from hard x-ray spectra as a function of sphere size.

enhancement of the field with spheres of sizes between 50 and $500 \mathrm{~nm}$ with the largest enhancement with $\sim 100 \mathrm{~nm}$ spheres, close to the size at which we observe the maximum hot electron yield. The fact that we observe the maximum electron enhancement for slightly larger spheres may be a result of the fact that even with the frequencydoubled light, some small preexpansion (a few tens of nm) of a target will occur prior to the main pulse arrival. Thus, $100 \mathrm{~nm}$ spheres may be so small that they do not survive completely in the rising laser field.

In addition to this Mie field enhancement, we also believe that multipass stochastic heating plays a role in producing greater numbers of hot electrons and higher $\mathrm{x}$-ray yields in our experiments. This multipass heating, 

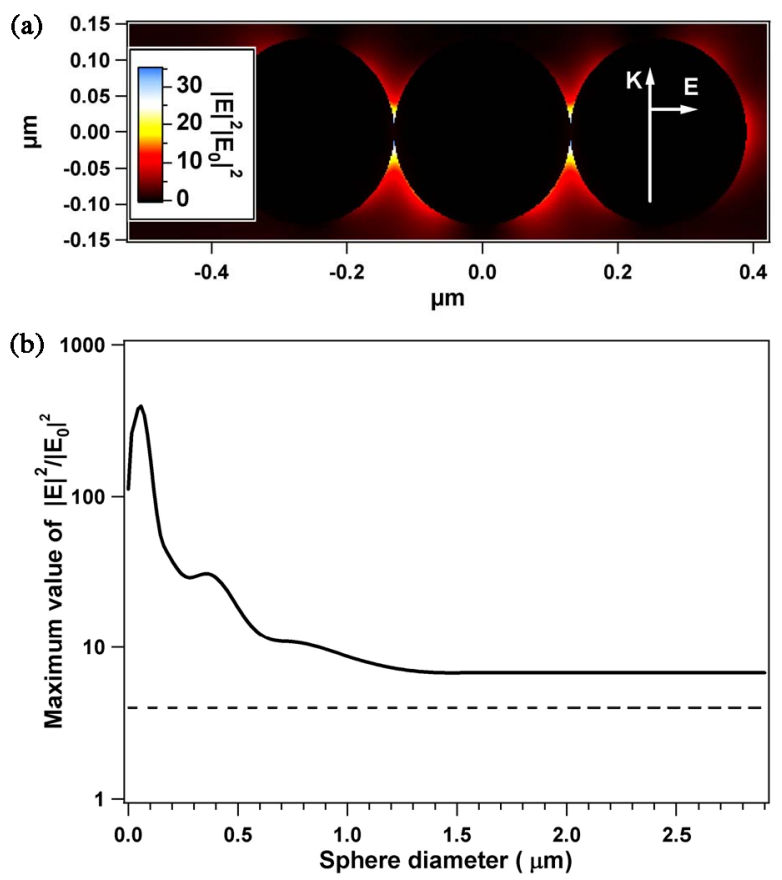

FIG. 4 (color online). a) Calculated field distribution around spheres on a surface arrayed in a hexagonal pattern for spheres with $260 \mathrm{~nm}$ diameter illuminated by $400 \mathrm{~nm}$ light. b) Calculated maximum enhancement of the square of the electric field when compared to vacuum fields as a function of sphere size irradiated. The dashed line illustrates the field that arises from $100 \%$ reflection from a planar surface.

first seen in particle-in-cell simulations [15] and since explored in more detail by Taguchi et al. [14] and Breizman et al. [13], will probably play a role in electron heating from these spheres. There are competing effects as the sphere size is increased. To undergo multiple passes in the plasma sphere, the electron must not be stopped in the plasma by collisions. In these polystyrene sphere plasmas, electrons with ponderomotive energy of $3 \mathrm{keV}$ (the ponderomotive potential of $400 \mathrm{~nm}$ pulses at $\sim 2 \times$ $10^{17} \mathrm{~W} / \mathrm{cm}^{2}$ ) will exhibit a stopping length of $200-$ $300 \mathrm{~nm}$ (using standard Spitzer-like mean-free-path formulae). Thus, spheres much larger than this size will inhibit multipass heating of the electrons because of collisional stopping. On the other hand, with smaller spheres, smaller volume and less efficient stochastic heating (see Ref. [12]) lead to lower hot electron production. It seems that these effects conspire with field enhancements generated by Mie resonances to produce an optimum in hot electron production with spheres of diameter $\sim 260 \mathrm{~nm}$.

In conclusion, we have presented an experimental study of the x-ray production from intense short pulse laser irradiation of solid targets coated with dielectric spheres of well-defined wavelength-scale sizes. We are able to enhance hot electron and $\mathrm{x}$-ray production from these targets by controlling the field structure through the introduction of the spheres on the target. By choosing spheres with diameter roughly half that of the laser wavelength, the combination of field enhancements from Mie resonances and efficient stochastic heating of the electrons leads to a substantial increase in hot electron number and a modest increase in electron temperature.

We would like to acknowledge the assistance of Jamie Landry and Gaurav Bansal in target preparation, Boris Breizman for theoretical insight, and Eckhart Foerster for loan and aid with the X-ray spectrometer. This work was supported by the National Science Foundation, the Welch Foundation Grant F-1626, the DOE Office of Fusion Energy Science under a Fusion Science Center, the National Nuclear Security Administration, and the Petroleum Research Fund.

[1] A. Rousse et al., Nature (London) 410, 65 (2001); C. W. Siders et al., Science 286, 1340 (1999).

[2] W.L. Kruer, The Physics of Laser Plasma Interactions, (Westview Press, Boulder, CO., 2003).

[3] F. Brunel, Phys. Rev. Lett. 59, 52 (1987).

[4] M. M. Murnane et al., Appl. Phys. Lett. 62, 1068 (1993); S. P. Gordon et al., Opt. Lett. 19, 484 (1994).

[5] G. Kulcsar et al., Phys. Rev. Lett. 84, 5149 (2000).

[6] C. Wulker, et al., Appl. Phys. Lett. 68, 1338 (1996).

[7] P. P. Rajeev et al., Phys. Rev. A 65, 052903 (2002).

[8] P. P. Rajeev et al., Phys. Rev. Lett. 90, 115002 (2003).

[9] T. Ditmire et al., Phys. Rev. A 53, 3379 (1996).

[10] H. M. Milchberg, S.J. McNaught, and E. Parra, Phys. Rev. E 64, 056402 (2001).

[11] G. Mie, Ann. Phys. (Berlin) 25, 377 (1908).

[12] D. R. Symes, A. J. Comley, and R. A. Smith, Phys. Rev. Lett. 93, 145004 (2004).

[13] B. N. Breizman, A. V. Arefiev, and M. V. Fomyts'kyi, Phys. Plasmas 12, 056706 (2005).

[14] T. Taguchi, T. M. Antonsen, and H. M. Milchberg, Phys. Rev. Lett. 92, 205003 (2004).

[15] P. Mulser, M. Kanapathipillai, and D.H.H. Hoffmann, Phys. Rev. Lett. 95, 103401 (2005).

[16] T. D. Donnelly et al., J. Phys. B 34, L313 (2001).

[17] H. Sumeruk et al., Bull. Am. Phys. Soc. 50, QP1.083 (2005).

[18] Y. B. Zel'dovich and Y. P. Raizer, Physics of Shock Waves and High Temperature Hydrodynamic Phenomena (Academic Press, New York, 1968), p. 258.

[19] D. W. Mackowski and M. I. Mishchenko, J. Opt. Soc. Am. A 13, 2266 (1996). 